\title{
LINGUISTIC THEORY AND MORPHOSYNTACTIC IMPAIRMENTS IN GERMAN AND ITALIAN APHASICS
}

\author{
Ria De Bléser**, Josef Bayer $\dagger$ and Claudio Luzzatti $\ddagger$ \\ *Cognitive Neurolinguistics, Potsdam University, F.R.G.; †Linguistics, University of Jena, F.R.G.; \\ $\ddagger$ Medical Psychology, Milan University, Italy
}

\begin{abstract}
At the turn of the century, the study of agrammatism started in German aphasiology with the introduction of syntax as an object of research in linguistics and the psychology of language. The disorder descriptively known as agrammatism was originally thought to arise from multiple origins, at the level of syntactic word order, of function word insertion, of grammaticalization by means of morphology, or as an impairment of processing. In modern aphasiology, there have been several attempts to reduce agrammatism to a single cause, such as the loss of specific syntactic operations or a deficit in morpholexical access. This article summarizes the results of morpholexical and morphosyntactic experiments with two German and two Italian agrammatic patients. The cross-language data reveal far more inflectional abilities than is usually assumed for agrammatism. Furthermore, they strongly support characterizations of the disorder as a syntactic rather than a morpholexical deficit.
\end{abstract}

\section{INTRODUCTION}

Any linguistic theory about the structure of words must account in one way or another for the fact that words have an internal as well as an external function. Morphemes are morphotactic in so far as they attach to other morphemes in order to allow the formation of a legitimate lexical item. They are syntactic in so far as they relate to an environment that lies beyond the word level. The internal aspect relates to the phonological side of morphology, the external aspect to the syntactic side. Morphology can thus be seen as an interface between the word and the phrase level of grammar.

There are various linguistic theories which differ with respect to the localization of morphology in a theory of grammar. The classical generative view is that derivational (and presumably compositional) morphology is a matter of the lexicon, while (regular) inflectional morphology is a matter of syntax [1]. In the late 1970s and early 1980s, this view was challenged by a strong version of lexicalism according to which syntactic processes cannot interfere with words, or more technically $\mathrm{X}^{\circ}$-categories. A recent version of such a theory is presented in di Sciullo and Williams [2]. Not only derivational but also inflectional morphemes would have to attach in a presyntactic component, and syntactic processes cannot analyse $\mathrm{X}^{\circ}$-structure. This view has been challenged, especially by Baker [3]. Baker argues that morphological processes are largely governed by syntactic principles such as Structure Preservation (see Emond's [4] Empty Category Principle (EC)) $[5,6]$, and that morphology is a set of well-formedness principles that supervise syntactic derivations which deal with processes that involve $\mathrm{X}^{\circ}$-categories. According to Baker's [3] and Chomsky's [6] theory, a verbal inflection is generated under a terminal

Address for correspondence: Prof. Dr. Ria De Bleser, Cognitive Neurolinguistics, Institute of Linguistics, University of Potsdam, PF 601553, D-14415, Germany 
syntactic node (called $\mathrm{I}^{\circ}$ for inflection). The complement of $\mathrm{I}$ is a VP. Since affixes cannot appear alone (they are bound elements), the verb, another zero-level category, moves and adjoins to I. Unfortunately, it is not quite clear from Baker's [3] elaboration what the status of classical derivational morphology is. Although it shares many properties with the morphosyntax of grammatical function changing, Baker's main topic, he still seems to consider a lexical status for it. *

With respect to linguistic processing, the first question is how close the processing system is to the grammar that is implemented. Assuming type-transparency, the three sketches of morphology make different predictions. According to the classical view, inflection and derivation should belong to syntactic and lexical processing, respectively. According to the lexicalist view, the processing of inflected forms should in principle be the same as the processing of derived forms. Both should be subject to mechanisms that are responsible for the comprehension and production of lexical items. Depending on one's assumptions about the architecture of the lexical morphological system, e.g. in the form of level ordering $[8,9]$, one would still expect differences between the processing of derived and inflected forms. These differences, however, could be traced back to the word level. However, if the process of word formation is subject to a generalized version of Move- $\alpha$, the processing of words should be similar to the processing of phrases in one important respect, namely, the binding of gaps. $\dagger$ Whereas this is imaginable on the basis of a complete parallelism of parser and grammar, it is unlikely that the formation of words ( $\mathrm{X}^{\circ}$-chains) should invoke the same processing operations as the formation of phrases involving XP-chains (Fig. 1).

\section{MORPHOLOGY AND APHASIA}

In the recent aphasiological literature, some evidence has been provided in favour of more or less explicit linguistic and/or processing models involving a morphological component. A proper evaluation of such evidence presupposes, however, that linguistic models or processing models show 'breakdown compatibility' [11]. Breakdown compatibility roughly means that the structure of the grammar/processor provides distinctions which can be correlated with neuropsychological dissociations. A system that does not allow for some isomorphism between components and neuropsychological dissociations is not breakdown compatible. Although our

*Take, however, the English affix -able. Its effect is clearly the same as PASSIVE. Thus, -able affixation is an operation of function changing. In Baker's system an example such as the washable book involves a D-structure the book wash-able [_] in which book receives its $\theta$-role from wash. Once wash moves to -able it loses its case-assigning property, and its object has to externalize. Notice that it is highly unclear in which sense an $\mathrm{X}^{\circ}$-category could be said to get case (see De Bleser and Bayer [7] for a critique of this assumption on the basis of German examples).

†Take as an example NP-movement. Bever and McElree [10] have shown that the human parser is sensitive to a gap (e, for "empty") as in:

(i) Bill $i$ was killed $\left[e_{i}\right]$.

Head-to-head movement is governed by the same mechanism in a representation like the one in (ii) which is German for "that Peter sings a song":

(ii) daß Peter [ $v P$ ein Lied $\left.e_{j}\right]$ [ $I$ sing $\left.i-t\right]$

that Peter a song sing-s.

Here, the verb has moved out of the VP into I where it adjoins to the inflectional head $-t$ ( 3 rd pers, sg.). For matters of processing the binding of the $X^{\circ}$-gap is, of course, a trivial matter because filler and gap are immediate neighbours. Relevant examples would have come from languages in which $\mathrm{X}^{\circ}$-incorporation moves some distance, e.g. in Oneida where we find, according to Baker [3], structures with 'possessor stranding' such as:

(iii) I house $e_{j}$-bought [NP John $e_{i}$ ]

"I bought John's House". 
Theory 1

classical

(Chomsky, 1970)
Theory 2

lexical morphology

(Kiparsky, 1982)
Theory 3

non-componential

morphology

(Baker, 1988)

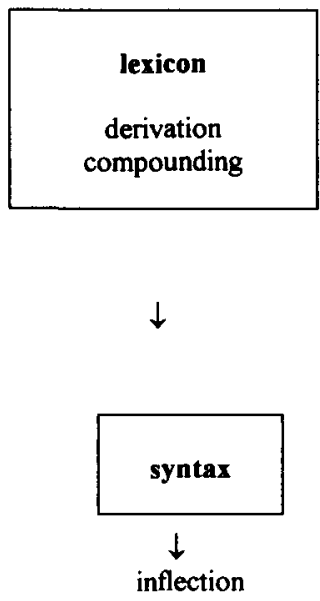

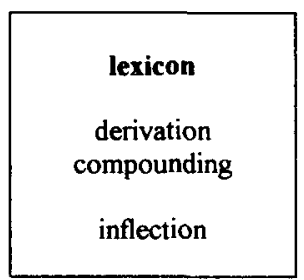

$\downarrow$

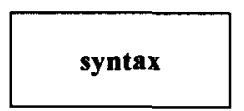

lexicon

idiosyncracies

Fig. 1. Linguistic models of the place of inflection in grammar.

present knowledge about such models is far too unspecific, a requirement like this seems to be called for if we want to see a relation between aphasic disorders and (psycho)linguistic models at all.

The aphasic disorder in which morphology was traditionally seen as most vulnerable is Broca's aphasia with agrammatic speech production.* In the spontaneous speech of Englishspeaking subjects of this kind, a surprising lack of functional elements can be observed. These elements include inflectional affixes as well as free function words such as pronouns, prepositions, and complementizers. Linguistic accounts of such aphasic deficits tried to define the class of missing elements as a natural category and to show that this category was inaccessible to the patients. Kean [13] gave a description of the deficit in terms of earlier phonological theory [14], according to which only minimal phonological words were retained in the output. Bradley et al. [15] argued that, in contrast to normals, agrammatic aphasics do not have a distinct access route for the function word vocabulary. The characterization of the deficit as a disturbed access route for functors was a refinement of the general idea of a central syntactic deficit [16].

As one shifts away from English, one can see that the status of morphology in agrammatic production cannot be as suggested by earlier work, namely, that bound morphemes (of a certain kind) are in principle inaccessible for the patient. Take the German verb, for example. The minimal phonological word is the stem which is mostly homophonouns with the imperative form; yet German patients overwhelmingly use the infinitive as a default form. The infinitive, however, is an inflected form consisting of stem $+e n$. Or take the more extreme case of Hebrew. The root morpheme of a Hebrew verb would be unpronounceable because it consists

*Meanwhile this clinical syndrome has been questioned or entirely rejected by a number of researchers [12] . 
only of a consonantal tier. Any form that can be pronounced requires the insertion of a vocalic tier. Thus, a morpheme stripping theory would not be applicable at all [11]. Meanwhile there are descriptions of data from a number of languages which uniformly show that agrammatic production does make use of morphology in general and inflectional morphology in particular $[17]$.

On the basis of in-depth studies of single cases, these global characterizations of the deficit have been subject to considerable rethinking and refinement. Owing to several factors, spontaneous production may not be a perfect mirror of the patient's linguistic competence. Patients may suffer from a variety of associate disorders such as dysarthria, phonological or speech apractic impairments, or memory disorders. Finally, one has to acknowledge that the ultimate goal of spontaneous speech is communication, i.e. conveying a message. In the face of a severe processing disorder, it is quite obvious that morphological and syntactic devices will be sacrificed in favour of the essential propositional structure of the utterance. However, the mere fact that certain features of a sentence are not produced does not necessarily mean that they are not available in the patient's linguistic system at all. They may be elicited under more constrained (experimental) tasks. Caramazza and Hillis [18], for example, studied an English-speaking patient who appeared fully agrammatic in her normal production. This patient (ML) was shown to handle the closed-class vocabulary (including inflectional morphemes) in tasks such as repetition, reading aloud, and writing to dictation, as long as they were restricted to the word level. In syntactic contexts the agrammatic picture reappeared in the same modalities, although the patient performed well on tasks such as sentence comprehension, grammaticality judgements and simple close tests in which she had to insert one of two (inflected) words into a syntactic frame.

In the following, we want to present results from a study in which German- and Italianspeaking patients with agrammatic language production and similar comprehension problems were compared. The results of the German investigation were published in Bayer et al. [19] and De Bleser and Bayer [20]. The Italian data are presented in detail in De Bleser and Luzzatti [21] and Luzzatti and De Bleser [22]. This article will summarize the main results for two German and two Italian patients who were maximally comparable according to an aphasia test (the Aachen Aphasia Test, AAT) which was standardized for the two languages $[23,24]$.

\section{SUBJECTS}

Two German patients, CB and MH, and two Italian patients, MG and DR, were selected for the cross-language morphosyntactic study at a time when their profiles on a standardized aphasia test (AAT) were practically identical. Their impairment was on the transition from a medium-to-mild degree of severity on the Token Test, the repetition tasks and the naming tasks, and they were all mildly impaired on the auditory and reading comprehension tasks. The two Italian patients ( $C R$ and $M G$ ) were also only mildly impaired on reading aloud and writing to dictation, whereas both German patients ( $C B$ and $M H$ ) showed a medium impairment on the parallel German tasks. All patients were right-handed, severely agrammatic in spontaneous speech and classified as clear cases of Broca's aphasia on the basis of the German and Italian AAT results, respectively $[23,24]$.

The first German patient, CB, was a 72-year-old former baker who had suffered a lefthemispheric stroke approximately 28 years before the present study. The CT-scan showed a large lesion in the entire territory of the middle cerebral artery with sparing only of the area 
subserved by the posterior temporal artery. CB's initial hemiplegia had almost perfectly recovered. His speech production was controlled and slowed down, severely agrammatic and characterized by word-finding problems. Communicative comprehension seemed to be fine. However, CB was severely impaired in the syntactic aspects of comprehension for both actives and passives.

The second German patient, $\mathrm{MH}$, was a 54-year-old female patient who had done secretarial work in her youth and had then become a housewife. Nine years earlier, at the age of 45 , she had also suffered extensive left-hemisphere damage in the entire territory of the middle cerebral artery. The initial global aphasia had recovered to a Broca's aphasia and by the time of the morphosyntactic testing, MH's speech production was also severely agrammatic but more fluent than that of CB and with more stereotypes and speech automatisms. She also showed syntactic comprehension problems for actives as well as passives, but they were milder than in CB.

The first Italian patient, DR, was a 27-year-old male dental technician who had suffered an aneurysm rupture 3 years earlier. The CT-scan showed a left-hemispheric subarachnoid haemorrhage and an intraparenchymatous satellite hematoma which was evacuated surgically. At the time of the morphosyntactic testing, the patient was still hemiplegic but the initial global aphasia had recovered to Broca's aphasia with clearly agrammatic speech. DR was also severely impaired in the syntactic aspects of comprehension for both actives and passives.

The second Italian patient, MG, had been run over by a truck at the age of 16 . Following neurosurgical treatment, the young man had a global aphasia with right-sided hemiplegia. The aphasia had recovered to the stage of a Broca's aphasia at the time of morphosyntactic testing. MG now had outspoken agrammatic speech production and he also showed syntactic comprehension problems for actives as well as passives, but they were milder than in DR. The CT-scan showed an extensive left-hemispheric cortical-subcortical fronto-central-parietal area of softening.

\section{EXAMINATION OF MORPHOLOGY IN ITS LEXICAL FUNCTION}

\section{Grammatical gender}

German has three grammatical genders, masculine, feminine and neutral. This feature must be encoded with the lexical entry of a given noun. In Italian, the noun-stem normally takes a suffix which determines the gender class. In this respect, Italian encodes gender formally in a sense that is not obvious in German.*

Patients were asked to supply the definite article to a given noun. This task is able to elicit the command of gender information inherent to a noun. The patients were given a noun which they had to repeat while adding the definite article. Table 1 summarizes the results.

The patients were also shown to be able to inflect correctly prenominal adjectives in German, and postnominal adjectives in Italian. $\dagger$

\section{Number}

German and Italian both mark the plural on the noun. While Italian has two different forms

\footnotetext{
*One could claim that bisyllabic nouns ending in -e are normally feminine. Since this -e deletes in certain word formation processes (Stube $\rightarrow$ Stübchen), one could argue that $-\mathrm{e}$ is a gender suffix. Notice that there are exceptions such as Käse $_{\text {masc. }}$ (= cheese) or Auge neuter $_{\text {(eye). }}$

†For Italian, nouns with regular and irregular suffixes were equally represented in this task, e.g. nas-o $o_{\text {masc. }}$ lung-o ("long nose") vs man- $o_{\mathrm{fem}}$ cald-a ("warm hand").
} 
Table 1. Gender assignment (\% correct)

\begin{tabular}{cccc}
\hline German & $N$ & CB & MH \\
& 60 & 93 & 93 \\
Italian & $N$ & DR* & MG \\
& 75 & 76 & 97 \\
\hline
\end{tabular}

*DR's difficulties in this task are confined to those items which deviate from the regular pattern according to which -o nouns are masculine and $-\mathrm{a}$ nouns are feminine. These include masculine and feminine nouns on $-e$ and also a small set of feminine nouns on $-o$ and masculine nouns on $-\mathrm{a}$.

(-e; -i) in addition to a zero-form and a number of exceptions such as uomo ("man') $\rightarrow$ uomini, German has about nine distinct plural forms.*

Patients were asked to pluralize a given singular noun and to singularize a given plural noun. As shown in Table 2, the patients were quite successful in this morphological task.

This investigation shows again that syntactically relevant inflectional morphology is in principle accessible to these agrammatic speakers. This impression has to be revised, however, when we begin to consider inflection with respect to its syntactic function.

\section{EXAMINATION OF MORPHOLOGY IN ITS SYNTACTIC FUNCTION}

In this section, we report a study of case morphology in German as well as a study of participle agreement in Italian.

\section{Morphological realization of abstract case in German}

In the case of an active transitive verb, there is an assignment of the accusative case to an object-NP and of the nominative case to the subject-NP. The former is assigned by the verb, the latter by the inflectional category I (inflection) which is attached to the verb. An abstract representation following the $X$-bar schema suggested by Chomsky [6] is given in Fig. 2 .

Figure 3 gives the underlying order of constituents in German, according to which $\mathrm{V}$ and I are in final position. German, however, has obligatory verb-second order in its root clauses. This order requires that the inflected verb is preposed and that some major constituent, not necessarily the subject-NP, is moved to the first position. An ordinary SVO sentence is derived from the presentation in Fig. 2 as shown in Fig. 3.

The position of the nominative-NP in Fig. 3 is a 'derived' position. In the same way as the

Table 2. Number conversion (\% correct)

\begin{tabular}{lccr}
\hline German & $N$ & CB & MH \\
Singular $\rightarrow$ plural & 90 & 79 & 86 \\
Plural $\rightarrow$ singular & 20 & 85 & 100 \\
& $N$ & DR & MG \\
Italian & 78 & 74 & 95 \\
Singular $\rightarrow$ plural & 78 & 82 & 96 \\
Plural $\rightarrow$ singular & 95 & 82 \\
\hline
\end{tabular}

\footnotetext{
*The suffix attached to a nominal stem of Italian carries not only number (singular or plural) but also gender information. Thus, for Italian, the nouns were given with the definite article in order not to confound the task with a test for gender.
} 


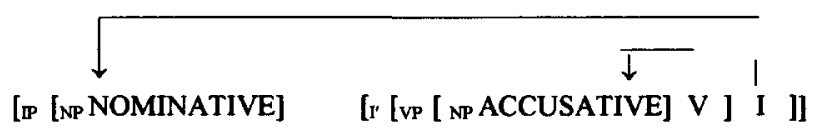

Figure 2: Underlying order of constituents in German. IP is short for inflectional phrase; it corresponds to the label for $\mathrm{S}$ (entence). The arrows show the processes of Case assignment.

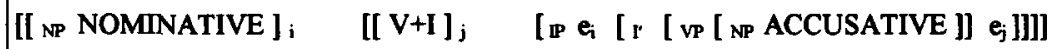

Figure 3: Verb-second order in German. $e_{i}$ and $e_{j}$ are empty positions which are "bound" by the displaced elements sharing an index.

nominative, the accusative-NP could have been moved there. This predicts that a sentence with the order NP V +I NP is potentially ambiguous with respect to the grammatical function of the NPs. In order to find out whether the first NP is an object, one has to look at the form of the second one.

The patients $\mathrm{CB}$ and $\mathrm{MH}$ were given the task of inflecting uninflected NPs in the context [_ $\mathrm{V}+\mathrm{I} \mathrm{NP}$ ] and [NP $\mathrm{V}+\mathrm{I}$ ] ] In order to do so, they had to scan the fully inflected NP because the missing case morphology was only recoverable on the basis of the complementary distribution between nominative and objective (accusative or dative) Case. As shown in Table 3, the patients were unable to deal with this task. Although the patients were able to supply a correctly inflected NP of the type DET $+A D J+N$, they had great difficulties in mapping the function of this morphology into a syntactically richer context.

Once the syntactic demand was reduced, the results improved. In Table 4, a similar experiment is reported in which case-inflection had to be supplied in the context of (a) a preposition (accusative or dative) and (b) another NP (genitive).

These results show that the patients must have an awareness of elementary syntactic relations

Table 3. Case assignment in subject- or object-NPs (\% correct)

\begin{tabular}{lccc}
\hline Type & $N$ & CB & MH \\
\hline Nominative & 20 & - & 30 \\
Accusative & 20 & $100^{*}$ & 30 \\
Dative & 20 & 15 & 5 \\
\hline
\end{tabular}

*This result cannot be taken at face value because of the patient's perseveration of the accusative. 
Table 4. Case-assignment for prepositional objects and possessor-NPs (\% correct)

\begin{tabular}{lccc}
\hline Type & $N$ & CB & MH \\
\hline $\begin{array}{l}\text { Preposition + accusative } \\
\text { Preposition + dative }\end{array}$ & 10 & 90 & $50^{*}$ \\
Genitive & 10 & 90 & 80 \\
\hline
\end{tabular}

*MH uses accusative throughout here, but never nominative.

as long as they are determined by a narrow enough context. Thus, they never insert a nominative in any of the prepositional objects. It is also remarkable that they can deal with the genitive case, which is not really frequent in NPs of this kind in colloquial German.

\section{Participle agreement in Italian}

Since Italian, unlike German, does not show overt case-marking in full NPs, it was necessary to investigate syntax-relevant morphology in another domain. An interesting and well-studied area to look at is the agreement of the past participle. The Italian past participle consists of the verbal root followed by the participle suffix $-t$, followed by an inflectional morpheme. The latter is in the unmarked, non-agreeing, form in $-o$, otherwise it is $-o,-a,-i$ or $-e$ depending on the features [ $\alpha$ gender, $\beta$ number]. Of syntactic interest is the fact that participle agreement correlates with the form of the auxiliary verb. Italian has two auxiliary verbs, essere (be) and avere (have). Avere is required for intransitive and active transitive verbs such as dormire (sleep) and mangiare (eat), while essere is required for ergative (unaccusative) verbs, such as r. rivare (arrive), cadere (fall), tornare (return), reflexive verbs like radersi (shave), lavarsi (wash oneself), and verbs in the passive voice. When a verb requires avere, there is no agreement between the subject-NP and the participle. When a verb requires essere, however, the participle agrees with the subject-NP. For avere there is one complication which was used in the experiments to be reported here: when the direct object of the verb is a clitic, which attaches the left of avere, the participle agrees with this clitic. Examples are given in Table 5.

Burzio [25] suggests as a rule of participle agreement that there must be a binding relation between an element (either the subject-NP or the direct object clitic) and the direct-object position of the verb. Ergative subjects, under this analysis, are generated as D-structure objects of the verb which get moved to their surface position.

From a processing point of view, participle agreement can only be performed successfully if there exists a lexical representation of verbs including their auxiliary. Apart from this, it is necessary to have a syntactic window that allows the joint representation of subject-NP or clitic object and the verbal participle.

Patients DR and MG were given sentences in which the participle appeared in the uninflected

Table 5. Auxiliary selection and participle agreement

(1) Maria ha vis-to due ragazzi Mary has seen two boys

(2) Maria è arriva-ta in ufficio Mary is arrived in (the) office

(3) Maria li ha vis-ti Mary them has seen 
form (vist_ instead of visto, vista, etc.). Their task was to supply the full form. The conditions were (a) non-agreement for avere, (b) subject-agreement for essere, (c) non-agreement with indirect-object clitic, (d) agreement with direct-object clitic. The results are presented in Table 6.

With one exception in the case of MG, these results show that the patients have a high sensitivity for the auxiliary verb as well as for the gender and number features of the antecedent with which the participle has to agree or not agree. In particular, the result on indirect-object clitics shows that the patients can well discriminate these from the direct-object clitics. MG, however, has problems with agreement with the direct-object clitics. In the cases in which he has incorrect responses, he either selects an unmarked form $(-o)$ or copies the feature from the subject position. One can explain this by the increased processing complexity which is required when agreement presupposes the distinction of alternative clitic forms, of which there are already nine for personal pronoun clitics. Otherwise, this task necessitates only limited syntactic processing abilities. The subject-NP as well as the clitic appear in immediate neighbourhood to the AUX/V-complex. In another task that was given to DR and MG this was not the case. Consider the following cases of an object-relative and coordination in Table 7.

In order to assign the correct participle agreement in such cases, it is necessary to parse the entire structure. In (1), the object-NP becomes available to the relative clause only via a coindexation between an empty operator before the che and a trace that relates it to the subjectposition of the relative clause. In (2), the parser has to detect a coordinate structure such that the participle will agree with the same NP as it does in the first conjunct.* In both cases there were no morphological cues on the basis of which the processing of the entire structure could be circumvented.

When given the same task in these syntactic environments, the performance of the patients dropped dramatically, as shown in Table 8. Obviously, the strategy was to match the participle agreement with one of the two antecedent NPs. The errors reflect a selection of the syntactically

Table 6. Participle agreement in simple clauses (\% correct)

\begin{tabular}{lcrr}
\hline Type & $N$ & DR & MG \\
\hline $\begin{array}{l}\text { Avere/non-agreement } \\
\begin{array}{l}\text { Essere/subject agreement } \\
\text { Indirect-object clitic/ }\end{array}\end{array}$ & 66 & 98 & 92 \\
$\begin{array}{l}\text { non-agreement } \\
\begin{array}{l}\text { Direct-object clitic/ } \\
\text { clitic agreement }\end{array}\end{array}$ & 80 & 100 & 100 \\
\hline
\end{tabular}

Table 7. Participle agreement in complex clauses: object relatives vs coordination

\begin{tabular}{|c|c|c|}
\hline (1) & $\begin{array}{l}\text { Il ragazzo } \text { è andato dalla nonna }_{j} \\
\text { The boy is gone to the grandmother }\end{array}$ & $\begin{array}{l}\text { che si èmmalat- } a_{j} \\
\text { who self is become ill (the grandmother is ill) }\end{array}$ \\
\hline (2) & $\begin{array}{l}\text { Il ragazzo } \text { ì andato dalla nonna }_{j} \\
\text { The boy is gone to the grandmother }\end{array}$ & $\begin{array}{l}\text { e si è ammalat- } 0_{i} \\
\text { and self is become ill (the boy is ill) }\end{array}$ \\
\hline
\end{tabular}

*The respective structures are given in $\left(1^{\prime}\right)$ and $\left(2^{\prime}\right)$ :

(1') [IP il ragazzo è andato dalla nonna $a_{j}$ [CP $\emptyset_{j}$ [C' che [IP $e_{j}$ si è ammalat-a]]]

(2') [IP [IP il ragazzo è andato dalla nonna] e [IP $\emptyset_{i}$ si è ammalat-o]]. 
Table 8. Participle agreement in complex clauses

(\% correct)

\begin{tabular}{lccc}
\hline Type & $N$ & DR & MG \\
\hline Relative clause & 16 & 69 & 38 \\
Coordination & 16 & 44 & 50 \\
\hline
\end{tabular}

inappropriate NP rather than a random selection of possible inflections.

The main generalization about these two sets of results is that the patients can exploit very limited syntactic contexts for the determination of agreement morphology, whereas they completely fail in more elaborate contexts. It thus cannot be the case that their morphological abilities are based on a fully operative syntactic competence. In cases where the patients fail, their responses still reflect (a) an intuition about legitimate word structures, and (b) the attempt to use morphology functionally, i.e. the forms which are selected have targets in the input even if they are the wrong targets.

\section{DISCUSSION}

Elicited data from severely agrammatic patients of two different languages have shown that agrammatism does not simply wipe out the ability to deal with morphology. It was demonstrated that these patients can deal quite successfully with inflectional morphology as long as it is determined by lexical information and a very limited syntactic context. Even this context was provided by the design of our experiments. In more complex tasks such as spontaneous production and certain comprehension tasks, it is thus no wonder that these patients use up their processing resources more rapidly than in the situation of elicitation. Given that they do not exploit inflectional morphology in less restricted situations, the question is why not?

The investigation presented above suggests that this cannot be due to the non-representation or non-accessibility of the morphological vocabulary. It rather seems to be the result of a lack of contexts in which it could be used. This view of the matter leads back to the models of morphology which were mentioned at the beginning of this article as well as the linguistic processor that may be connected to them. Of course, we are aware of the fact that neurolinguistic data like those presented here cannot be conclusive with respect to the choice of linguistic models. However, they are suggestive in the sense that the use of inflectional morphology does not seem to presuppose a full-fledged syntactic system as was proposed by the classical model. Since Baker [3] mainly focuses on function-changing morphology, which he takes to be guided by syntactic principles, it is difficult to say how inflectional morphology would fall into place. It is also unclear how a processing system would implement the operations of head-to-head movement and incorporation. Thus, our data have little or nothing to say about such a model.

With respect to lexical morphology, things are different. Here, the representational system itself reflects the autonomy of word-level operations irrespective of their function in syntax. A processor that would implement such a grammar would presumably involve a morphological parser that decides on the well-formedness of an $\mathrm{X}^{\circ}$-item. The rules and principles that it uses would be largely independent of the phrase-building system and the modules which license case, $\theta$-roles, syntactic gaps, etc. The data presented above are compatible with such a grammar/processor because the linguistic breakdown observed follows its global architecture.

One may ask why the German patients appear to be less able to deal with syntactic-relevant 
morphology than the Italian patients, although the Italian patients were quite comparable otherwise. Recall that $\mathrm{CB}$ and $\mathrm{MH}$ already had difficulties in providing case morphology in simple SVO clauses, whereas DR and MG had little difficulties with participle agreement in simple sentences. Upon closer linguistic scrutiny, however, it turns out that there are languagespecific reasons for this difference. As we have shown in Fig. 3, what appears to be an SVO sentence in German is the result of verb movement and topicalization of the subject-NP. According to this analysis, the subject is in a derived position. In its place one could as well have a non-subject phrase. This may lead to a complexity that is not found in Italian, which is already underlying an SVO language. The preverbal position in Italian is much more likely to contain the subject such that auxiliary and verbal agreement can rely on positional information. This is but one example which shows that a linguistic analysis of the materials to be used in cross-language aphasiology is indispensable.

\section{REFERENCES}

1. Chomsky, N. Remarks on nominalization. In Readings in English Transformational Grammar, R. Jacobs and P. Rosenbaum (Editors). Mouton, The Hague, 1970.

2. Sciullo, A. di and Williams, E. On the Definition of Word. MIT Press, Cambridge, MA, 1987.

3. Baker, M. C. Incorporation. A Theory of Grammatical Function Changing. University of Chicago Press, Chicago, 1988.

4. Emonds, J. A Transformational Approach to English Syntax: Root Structure-preserving and Local Transformations. Academic Press, New York, 1976.

5. Chomsky, N. Lectures on Government and Binding. Foris, Dordrecht, 1981.

6. Chomsky, N. Barriers. MIT Press, Cambridge, MA, 1986.

7. De Bleser, R. and Bayer, J. German word formation and aphasia. Linguistic Review 6, 1-40, 1986.

8. Kiparsky, P. Lexical morphology and phonology. In Linguistics in the Morning Calm, Linguistic Society of Korea (Editor). Hansin, Seoul, 1982.

9. Halle, M. and Mohanan, K. P. Segmental phonology of English. Linguistic Inquiry 16, 57-116, 1985.

10. Bever, T. and McElree, B. Empty categories access their antecedents during comprehension. Linguistic Inquiry 19, 35-43, 1988.

11. Grodzinsky, Y. The syntactic characterization of agrammatism. Cognition 16, 99-120, 1984.

12. Badecker, W. and Caramazza, A. On considerations of method and theory governing the use of clinical categories in neurolinguistics and cognitive neuropsychology. Cognition 5, 9-46, 1985.

13. Kean, M. L. The linguistic interpretation of aphasic syndromes: agrammatism in Broca's aphasia, an example. Cognition 5, 9-49, 1977.

14. Comsky, N. and Halle, M. The Sound Pattern of English. Harper and Row, New York, 1968.

15. Bradley, D. C., Garrett, M. F. and Zurif, E. B. Syntactic deficits in Broca's aphasia. In Biological Studies of Mental Processes, D. Caplan (Editor). MIT Press, Cambridge, MA, 1988.

16. Berndt, R. and Caramazza, A. A redefinition of the syndrome of Broca's aphasia: implications for a neuropsychological model of language. Applied Psycholinguistics 1, 225-278, 1980.

17. Menn, L. and Obler, L. K. Agrammatic Aphasia. A Cross-Language Narrative Sourcebook, Vols 1-3. John Benjamin. Amsterdam, 1990.

18. Caramazza, A. and Hillis, A. The disruption of sentence production: a case of selective deficit to positional level processing. Brain and Language 36, 365-650, 1989.

19. Bayer, J., De Bleser, R. and Dronsek, C. Form und Funktion von Kasus bei Agrammatismus. In Grammatik und Kognition, J. Bayer (Editor). Westdeutscher Verlag, Wiesbaden, 1987.

20. De Bleser, R. and Bayer, J. On the role of inflectional morphology in agrammatism. In Theoretical Morphology, M. Hammond and M. Noonan (Editors). Academic Press, New York, 1988.

21. De Bleser, R. and Luzzatti, C. Morphological processing in Italian agrammatic speakers: syntactic implementation of inflectional morphology. Brain and Language 46, 21-40, 1994.

22. Luzzatti, C. and De Bleser, R. Morphological processing in Italian agrammatic speakers. Lexical morphology. Brain and Language (in press).

23. Huber, W., Poeck, K., Weniger, D. and Willmes, K. Der Aachener Aphasie-Test. Hogrefe, Göttingen, 1983.

24. Luzzatti, C., Willmes, K. and De Bleser, R. Aachener Aphasie Test (AAT): Versione Italiana. Organizzazione Speciali, Firenze, 1991.

25. Burzio, L. On Italian Syntax. A Government-binding Approach. Reidel, Dordrecht, 1986. 